\title{
Rejeição entre pares - diálogo com o filme The Perks of Being a Wallflower
}

\section{Peer rejection - dialogue with the movie The Perks of Being a Wallflower}

\section{Rechazo entre iguales - diálogo con la película The Perks of Being a Wallflower}

\author{
Sarah Izbicki* \\ Universidade de São Paulo - USP, São Paulo, São Paulo, Brasil \\ Luiza Chagas Brandão** \\ Universidade de São Paulo - USP, São Paulo, São Paulo, Brasil \\ Lígia Mosolino de Carvalho*** \\ Universidade de São Paulo - USP, São Paulo, São Paulo, Brasil \\ Márcia Helena da Silva Melo**** \\ Universidade de São Paulo - USP, São Paulo, São Paulo, Brasil
}

\begin{abstract}
RESUMO
A rejeição entre pares é uma categoria sociométrica que revela baixa preferência no grupo de colegas. A ela pertencem crianças que recebem muitas nomeações negativas e poucas nomeações positivas de seus pares, quando comparadas com a média do grupo. Apesar de ser um problema corrente na infância e relativamente estável, com o tempo, além de se relacionar com delinquência juvenil, abuso de substâncias, evasão escolar e depressão, é um tema pouco investigado e abordado no Brasil. Neste contexto, este artigo teve por objetivo salientar características importantes sobre a rejeição entre pares relatadas na literatura, relacionando este status sociométrico com a amizade estabelecida com colegas, os comportamentos agressivos que podem ser apresentados pela criança rejeitada, suas habilidades sociais e o papel do professor neste contexto. Como forma de dialogar com os aspectos descritos e exemplificá-los, utilizou-se o filme The perks of being a wallflower, por aproximar a questão às interações cotidianas de maneira dinâmica e interativa. Espera-se que o uso do filme como recurso motivacional e didático, aumente a visibilidade do tema no meio acadêmico e entre as pessoas que se constituem como figuras responsáveis pelo desenvolvimento saudável da criança, inclusive no que diz respeito à rejeição pelos pares.

Palavras-chave: rejeição entre pares, amizade, agressividade, habilidades sociais, interação professor-aluno.
\end{abstract}

\section{ABSTRACT}


Peer rejection is a sociometric category that reveals a low preference among the peer group. To this category belong children that receive a lot of negative nominations and a few positive ones from their peers, when compared with the group average. Besides being a common problem at childhood and relatively stable over time, and also being related to juvenile delinquency, drug abuse, scholar evasion and depression, it is a theme not very studied in Brazil. This article's objective was to underline some rejection's important characteristics described at PSI the literature, relating this sociometric status with peer friendship, social abilities and teacher's role in this context. The movie The Perks of Being a Wallflower was used to exemplify and dialogue with the described aspects. Using the movie as a motivational and didactic resource, the objective was to enhance the visibility of this subject both in the academic field and in the group of people responsible for child development.

Key-words: peer rejection, friendship, aggressive behavior, social skills, teacher student interaction.

\section{RESUMEN}

El rechazo entre iguales es una categoría sociometrica que revela baja preferencia en el grupo de colegas. A ella pertenecen niños que reciben muchas nominaciones negativas y pocas nominaciones positivas de sus iguales, cuando en comparación con la media del grupo. A pesar de ser un problema corriente en la infancia y relativamente estable con el tiempo, además de relacionarse con delincuencia juvenil, abuso de sustancias, evasión escolar y depresión, es un tema poco estudiado en Brasil. Este artículo tuvo por objetivo destacar características importantes del rechazo entre iguales descritas en la literatura PSI, relacionando este status sociométrico con la amistad establecida con colegas, las conductas agresivas que el niño rechazado puede presentar, sus habilidades sociales y el papel del maestro en este contexto. Como forma dialogar con los aspectos descritos y ejemplificarlos, fue utilizada la película The perks of being a wallflower, por acercar la cuestión a las interacciones cuotidianas de manera dinámica y divertida. Esperase que la utilización de la película como recurso motivacional y didáctico aumente la visibilidad del tema en el medio académico y entre las personas responsables por el desarrollo saludable del niño.

Palabras-clave: rechazo entre iguales, amistad, agresividad, habilidades sociales, interacción maestro-alumno.

\section{Introdução}

No contexto escolar, as interações estabelecidas com os pares exigem do indivíduo a aquisição de habilidades motoras, linguísticas e afetivas específicas (Castro, Melo, \& Silvares, 2003; Z. Del Prette \& Del Prette, 2013). Com base no êxito ou no fracasso dessas relações, os colegas desenvolvem percepções em torno das crianças que se configuram como importantes fatores para o desenvolvimento do indivíduo ao impactarem sua autoavaliação e reforçarem determinadas condutas (Dijkstra, Lindenberg, \& Veenstra, 2007; Veenstra, Lindenberg, Munnisksma, \& Dijkstra, 2010). 
A percepção que os pares têm sobre uma criança pode refletir em seu status sociométrico, ou seja, o quanto essa criança é incluída ou excluída socialmente. São vários os procedimentos utilizados na investigação desse status, sendo que a avaliação sociométrica por nomeação é o mais utilizado (Silvares \& Melo, 2010; Fink, Rosnay, Peterson, \& Slaughter, 2013). Neste procedimento, as crianças de um determinado grupo são solicitadas a nomear certo número de colegas com quem mais gostam (nomeações positivas) e menos gostam (nomeações negativas) de brincar ou trabalhar. A partir das respostas fornecidas, têm-se indícios de níveis de aceitação e rejeição pelo grupo (Coie, Dodge, \& Coppotelli, 1982).

Vários estudos descrevem diferentes tipos de categorias às quais a criança pode pertencer, segundo a preferência de seus pares, obtidas a partir de seus níveis de aceitação e rejeição social. Utilizando medidas do impacto (o número de nomeações positivas mais o número de nomeações negativas) e da preferência social (o número de nomeações positivas menos o número de nomeações negativas), pode-se chegar a categorias avaliativas, tais como as descritas por Coie et al. (1982), a saber, 1) Popular: refere-se à criança altamente preferida pelos pares, com grande número de nomeações positivas e baixo número de nomeações negativas; 2) Rejeitada: refere-se à criança que não é querida em seu grupo, ou seja, com elevado número de nomeações negativas e baixo número de nomeações positivas; 3) Negligenciada: relaciona-se à criança que não recebe nomeações positivas, apresentando também baixo ou nenhum número de nomeações negativas; 4) Controversa: relaciona-se à criança que gera sentimentos ambivalentes em seu grupo, ou seja, com grande número de nomeações positivas e negativas por seus pares e 5) Mediana: referente à criança que recebe um número de nomeações (tanto positivas como negativas) dentro da média do grupo. As categorias anteriores representam padrões de interação que se sobressaem em relação à média.

A mais estável das categorias sociométricas propostas por Coie et al. (1982) é a referente às crianças rejeitadas. Estas crianças são as que pertencem ao grupo de risco para delinquência juvenil, abuso de substâncias, evasão escolar e depressão - relação que não se apresenta nas demais categorias (McFadyen-Ketchum \& Dodge, 1998; Mrug et al., 2012). A rejeição entre pares é um problema comum na infância, sendo que aproximadamente $12 \%$ das crianças atingem critérios sociométricos que as enquadram nessa categoria (McFadyen-Ketchum \& Dodge, 1998; Melo, 2006; Silvares \& Melo, 2010). Dada a alta incidência de rejeição infantil entre os pares e suas consequências negativas para a criança rejeitada, vários estudos referentes a esse status social têm sido conduzidos, com o intuito tanto de conhecer suas especificidades quanto de investigar maneiras de reduzir a rejeição pelos colegas (Lansford, Dodge, Fontaine, Bates, 
\& Pettit, 2014; Leflot, van Lier, Onghena, \& Colpin, 2013; Mikami, Lerner, \& Lun, 2010; Mrug et al., 2012).

Não existe um único perfil de crianças rejeitadas, mas existem determinados padrões comportamentais relacionados à rejeição. Segundo Mikami et al. (2010), diversos autores citam como exemplos desses padrões, comportamentos agressivos mal-adaptativos, comportamentos retraídos, dificuldades na regulação emocional e baixa frequência de emissão de comportamentos prossociais. Destaca-se que o contexto social no qual ocorre a interação com pares parece ter uma importante influência na rejeição (Mikami et al., 2010; Scott, 2015), uma vez que é necessário que sejam identificadas as variáveis ambientais funcionalmente relacionadas com as variáveis do organismo para se compreender inteiramente o modo pelo qual tais relações são estabelecidas, mantidas e modificadas nas interações organismo-ambiente.

A partir de uma rápida busca em duas importantes bases de dados na área da psicologia, realizada em julho de 2014, encontraram-se 786 artigos internacionais utilizando os descritores "peer AND rejection" no resumo na PsycINFO, ao passo que foram localizados apenas seis artigos brasileiros com os descritores "rejeição AND pares", também no resumo, na Scielo (Scientific Electronic Library Online). Com isto, nota-se que, enquanto a rejeição por pares tem sido vastamente investigada internacionalmente, ela tem sido pouco estudada no Brasil, a despeito da sua grande relevância. Desta forma, tornam-se necessários esforços na direção de aumentar a visibilidade deste tema tanto no meio acadêmico, propiciando mais estudos na área, quanto entre as pessoas que estão em contato constante com crianças e adolescentes, tais como pais e professores, os quais têm um importante papel na prevenção e na remediação da rejeição e dos problemas a ela associados.

Tendo em vista os pontos acima destacados, este artigo busca descrever importantes aspectos envolvidos na temática da rejeição por pares, além de ilustrá-los com base no filme The perks of being a wallflower. Especificamente, tem-se por objetivo explorar a influência das amizades, uma vez que esta parece ter significativa importância tanto para a prevenção ou manutenção do status de rejeição quanto para o modo como ele afetará a vida do indivíduo rejeitado; discutir os dois padrões comportamentais da criança rejeitada considerados mais relevantes pela literatura, a saber: o déficit nas habilidades sociais; a agressividade; e a abordagem sobre o papel do professor na rejeição entre pares, uma vez que os docentes exercem considerável influência no relacionamento entre os alunos e podem contribuir com a prevenção da rejeição e suas consequências negativas. 


\section{Método}

Os principais temas relacionados, pela literatura da área, à rejeição de pares, e acima listados, foram descritos e, em seguida, exemplificados com características e cenas vividas pela personagem central do filme The perks of being a wallflower: Charlie. O uso de um longa-metragem como material ilustrativo permite uma aproximação dinâmica e interativa entre as relações cotidianas vivenciadas pelas crianças e adolescentes, de um lado e, de outro, o tema da rejeição entre pares, de modo a despertar o interesse sobre o assunto (Farias \& Ribeiro, 2007).

O filme, dirigido por Stephen Chbosky, é uma adaptação de seu livro homônimo. Foi lançado em 2012 nos Estados Unidos e, ao ser lançado no mesmo ano no Brasil, recebeu o nome de "As vantagens de ser invisível". Nele, é narrada a história de Charlie, um adolescente americano de 15 anos de idade, iniciando o Ensino Médio em uma nova escola, na qual enfrenta dificuldades para se adaptar e fazer amigos. Sua vida é marcada por uma série de eventos estressantes que vão sendo revelados ao longo do filme, tais como um abuso sexual na infância, o suicídio de seu melhor amigo e um transtorno mental - não especificado no filme - que levou a internações psiquiátricas. São retratados a entrada de Charlie em um grupo de amigos e o seu relacionamento com esses pares, caracterizado tanto pelo acolhimento que recebe destes quanto pelas dificuldades enfrentadas no grupo. O filme conta também como ele desenvolve uma relação especial com seu professor de literatura, Mr. Anderson, que incentiva seu interesse pela leitura e escrita e conversa com Charlie sobre algumas dificuldades vivenciadas por ele.

Apesar de não ser possível afirmar categoricamente que Charlie é rejeitado pelos seus pares no Ensino Médio, ou que foi rejeitado em seus primeiros anos escolares (para isso, seria necessária uma avaliação sociométrica com seus colegas), pode-se afirmar que ele apresenta uma série de características comumente relacionadas à rejeição. Por este motivo, o filme auxilia na caracterização de aspectos associados à criança rejeitada, em termos tanto de seus comportamentos quanto da importância das relações estabelecidas entre a criança e aqueles que estão a sua volta.

Vale mencionar que, por se tratar de um filme, tem-se acesso apenas a recortes das interações das personagens, havendo informações limitadas sobre as variáveis que controlam o comportamento das pessoas retratadas no filme (Farias \& Ribeiro, 2007). Por esta razão, salienta-se que as referências ao filme realizadas no presente trabalho visam à ilustração dos temas expostos, não se tratando de uma análise de seu enredo e de suas cenas baseada nos conceitos inerentes ao tema da rejeição social. 
Deve-se ressaltar, por fim, que, ainda que Charlie seja adolescente, optou-se por utilizar o filme para exemplificar os aspectos mais importantes associados à rejeição na infância, pois, além do status da rejeição ser relativamente estável com o tempo (Cillessen, 2009; Cillessen, Bukowski, \& Haselager, 2000), o filme indica que Charlie manteve o mesmo estilo comportamental apresentado na infância, o que sugere que ele foi rejeitado quando criança.

\subsection{Amizade e rejeição}

Ser rejeitado pelos pares é um fenômeno de grupo, enquanto ter amigos se refere a um nível individual. A partir de dados obtidos por meio da avaliação sociométrica, a amizade é definida pela reciprocidade na nomeação positiva entre crianças (Hodges, Malone, \& Perry, 1997; Parker, 2011). Crianças mais aceitas pelos colegas tendem a se envolver mais em amizades diádicas do que as crianças menos aceitas, e esta disparidade tende a ser maior para meninos do que para meninas. Apesar dessa tendência, nem toda criança bem aceita pelo grupo de pares tem amigos mútuos, e nem todas as crianças rejeitadas carecem de amizade recíproca (Parker \& Asher, 1993).

Estudos (Bagwell \& Schmidt, 2013; Laursen, Bukowski, Aunola, \& Nurmi, 2007; Mikami \& Normand, 2015) indicam que ter amigos recíprocos minimiza algumas consequências adversas relacionadas à rejeição social e às dificuldades individuais de ajustamento. Entre crianças sem amigos, a rejeição por pares foi associada a aumentos subsequentes de problemas de comportamento internalizantes (que se expressam em relação ao próprio indivíduo, tais como o comportamento ansioso e o comportamento depressivo) e externalizantes (que se expressam em relação a outras pessoas, tais como o comportamento agressivo e o comportamento de oposição), enquanto esta associação não foi feita para crianças com amigos. Ao mesmo tempo, esses dois tipos de problemas de comportamento foram preditores de aumentos subsequentes na rejeição por pares. Portanto, entende-se que os problemas relacionais com os colegas e os problemas de ajustamento se retroalimentam, sendo a amizade uma variável crítica nesse processo cíclico (Laursen et al., 2007).

Problemas de comportamento internalizantes ou externalizantes são mais fortemente relacionados à vitimização ${ }^{1} \mathrm{em}$ crianças rejeitadas pelos seus pares ou carentes de amigos recíprocos, com amigos incapazes de cumprir uma função de proteção (p. ex. pares fracos fisicamente), quando comparadas a crianças que possuem melhores amigos, amigos capazes de defendê-los, ou que são melhor apreciadas pelo grupo de colegas (Hodges et al., 1997). Estudos indicam que a vitimização está relacionada ao aumento de problemas de comportamento somente para crianças sem um amigo mútuo 
(Bagwell, Kochel, \& Schmidt, 2015; Hodges, Boivin, Vitaro, \& Bukowski, 1999), de forma que a amizade teria uma função protetora na diminuição do risco de vitimização. Ademais, crianças sem amizade recíproca tendem a ser mais solitárias do que crianças com amigos, independentemente do quão bem aceitas elas são pelo grupo de pares (Parker \& Asher, 1993).

A solidão é a primeira característica marcante de Charlie, apresentada no início do filme com uma carta escrita pelo protagonista, na qual relata este sentimento: passara todo o período de férias escolares de verão sem conversar com outras pessoas que não sua família e, com a proximidade da volta às aulas, escreve "Só espero que eu faça um amigo". No início desse novo período escolar, ele tenta passar despercebido pelos corredores, almoça sempre sozinho e pessoas que anteriormente o conheciam fingem não 0 conhecer. Seu único amigo, Michael, suicidara-se no período escolar anterior.

O próprio termo em inglês "wallflower", utilizado para caracterizar o protagonista no título do filme, refere-se à solidão e ao isolamento social. "Wallflower" é um termo que pode ser utilizado para nomear um indivíduo que não se socializa ou participa de atividades ou eventos sociais. De acordo com o Oxford English Dictionary (1989), essa palavra, ainda que fosse originalmente usada para se referir a mulheres que, no contexto de bailes, mantinham-se isoladas na parede do salão, teve sua definição expandida para se referir também a homens em contextos sociais diversos. Esta expressão não possui uma tradução direta para o português, e a escolha do termo "invisível" não parece refletir adequadamente a situação de Charlie em sua escola, uma vez que ele não é "invisível", no sentido de não ser notado pelos colegas, e sim apresenta dificuldades em interações sociais. Deste modo, optou-se por utilizar neste artigo o título no idioma original do filme, uma vez que "wallflower" retrata mais adequadamente o comportamento de Charlie no início do filme, quando ainda não possui nenhum amigo e, tal como indicado pela definição encontrada no dicionário, apresenta dificuldades em interagir com outras pessoas em uma festa, permanecendo isolado, próximo à parede.

A amizade que Charlie acaba fazendo ao longo do filme com um grupo de jovens desviantes das normas sociais dos grupos populares da escola atenua sua condição de solidão e outros comportamentos internalizantes. Não apenas a presença dessas amizades reduz os problemas vivenciados pelo protagonista, como também, em dois momentos do filme em que o os amigos do protagonista se afastam, seus problemas internalizantes retornam e em maior intensidade, voltando a melhorar com a reaproximação dos amigos.

Embora as qualidades positivas das amizades de jovens rejeitados possam compensar, em certa medida, seu status de rejeição e sua 
solidão, é frequente a associação desses jovens com pares desviantes, podendo promover ou intensificar o desenvolvimento de comportamentos antissociais, tais como: a agressividade, o uso de álcool e de substâncias psicoativas (Monahan, Steinberg, \& Cauffman, 2009; Salazar et al., 2015).

Este outro aspecto relacionado à amizade entre jovens rejeitados também é apresentado no filme, uma vez que Charlie, antes de se unir a seu novo grupo de amigos, nunca havia usado drogas e, possivelmente, não consumia bebidas alcoólicas ou frequentava festas noturnas, o que pode ser deduzido pela sua referência ao fato de que o pai de seu amigo Michael era alcoolista e que, por isso, não gostava de bebidas ou festas. Após a associação com os novos amigos, passa a ser frequente a ida a festas nas quais os convidados consumiam bebidas alcoólicas e drogas, marcando o início de Charlie no uso de tais substâncias.

\subsection{Rejeição e habilidades sociais}

Podem-se definir habilidades sociais como classes de comportamentos sociais que fazem parte do repertório do indivíduo e permitem a ele enfrentar adequadamente demandas advindas de situações interpessoais (Argyle \& Furnham, 1981; Z. Del Prette \& Del Prette, 1999; Z. Del Prette \& Del Prette, 2010). Esses comportamentos podem ser divididos em sete categorias: autocontrole e expressividade emocional, habilidades de civilidade, empatia, assertividade, solução de problemas interpessoais, fazer amizades e habilidades sociais acadêmicas, sendo que cada uma dessas é composta de diferentes subclasses ( $Z$. Del Prette \& Del Prette, 2005). No contexto escolar, as habilidades sociais mais enfatizadas e valorizadas nos estudos internacionais dos últimos anos, segundo metanálise da literatura (Caldarella \& Merrell, 1997), podem ser agrupadas em cinco conjuntos de comportamentos: (1) relação com os companheiros (cumprimentar, elogiar, oferecer ajuda ou assistência, convidar para jogo de interação); (2) autocontrole (controlar o humor, seguir regras, respeitar limites); (3) habilidades sociais acadêmicas (envolver-se na tarefa, realizá-la de forma independente, seguir instruções); (4) ajustamento (seguir regras e comportar-se de acordo com o esperado); e (5) asserção (iniciar conversação, aceitar elogios, fazer convites). A competência social, por sua vez, relaciona-se com a adequação do comportamento social a seu contexto, devendo, portanto, ser modelada e avaliada de acordo com a comunidade verbal na qual o indivíduo está inserido ( $Z$. Del Prette \& Del Prette, 2010).

A rejeição se relaciona à falta de competência social, e não necessariamente à ausência de uma ou mais habilidades sociais (Bierman, 2004; Z. Del Prette \& A. Del Prette, 2010). Esse status 
sociométrico pode ser ocasionado pela dificuldade do indivíduo em interagir adequadamente com a comunidade na qual está inserido. Ser rejeitado, por sua vez, priva a criança de uma série de situações nas quais ela poderia treinar e desenvolver habilidades sociais. Desta maneira, forma-se um círculo vicioso no qual o desenvolvimento de habilidades e competências sociais fica prejudicado, contribuindo para a manutenção do status da rejeição (Mrug et al., 2012).

Em termos das habilidades sociais de Charlie, o filme mostra a preocupação do personagem, antes e durante o primeiro dia de aula, em fazer um amigo e "em fazer com que as coisas sejam diferentes nesse novo ano", o que sugere o quanto, nos anos anteriores, ele apresentou dificuldades em se relacionar com seus colegas. Essas cenas indicam um possível déficit de Charlie na categoria "fazer amigos", referida por Z. Del Prette e A. Del Prette (2005). O filme demonstra também situações nas quais o protagonista deixa de ter acesso a possíveis reforçadores sociais por falta daquilo que esses autores denominam de "assertividade". Por exemplo, são apresentadas as dificuldades pelas quais Charlie passa por não ser assertivo com sua namorada, ao não conseguir mostrar seu desejo de não estar mais com ela e, assim, não poder vivenciar um relacionamento mais reforçador com outra pessoa.

A falta de competência social, pela sua própria definição, é mais difícil de ser exemplificada, uma vez que depende da avaliação dos membros do grupo em que o indivíduo está inserido. Porém, há uma situação no filme que deixa clara a avaliação negativa do grupo ao comportamento de Charlie: durante um jogo de "verdade ou desafio", no qual ele é desafiado a dar um beijo na garota mais bonita do grupo, ele beija sua melhor amiga ao invés de sua namorada, que está sentada ao seu lado. O grupo reage olhando de maneira incriminadora para Charlie e prestando apoio emocional a sua namorada, que ficou abalada com a resposta dele. Após este episódio, Charlie passa a ser excluído também por seus amigos, 0 que exemplifica a ideia de que a avaliação que o grupo faz dos comportamentos dos pares (no caso do filme, o comportamento de Charlie ao beijar outra menina, e não sua namorada) é mais importante do que a falta de habilidades sociais em si (tal como sua falta de assertividade) para o estabelecimento da condição de rejeição, conforme Bierman (2004) e Z. Del Prette e Del Prette (2010).

\subsection{Rejeição e agressividade}

Diversos estudos têm estabelecido relações entre a rejeição social pelos pares e a agressividade (e.g. Orue \& Calvete, 2011; López, Olaizola, Ferrer, \& Ochoa, 2006; Newcomb, Bukowski, \& Pattee, 1993). Inicialmente, as pesquisas se focavam nos altos índices de 
comportamento agressivo apresentado pelas crianças rejeitadas, mas pesquisas posteriores passaram a sugerir que nem todas as crianças rejeitadas são agressivas e nem todas as crianças agressivas são rejeitadas (López et al., 2006). Aproximadamente metade dos alunos rejeitados apresenta um perfil de comportamento agressivo, ao passo que a outra metade é caracterizada pela dificuldade em outras habilidades sociais, apresentada como comportamentos passivos e de timidez, não exibindo comportamentos agressivos no contexto escolar (López et al., 2006).

São vários os fatores que podem determinar se a criança agressiva será rejeitada. Os estudos dão grande importância às normas do grupo social ao qual a criança pertence, no sentido de que comportamentos sociais negativos - tal como a agressão -, quando não são frequentes no grupo, levam com maior probabilidade a avaliações negativas pelos pares. Mais do que isso, sugere-se que não é o comportamento da sala como um todo que determina a avaliação social da criança, mas sim o comportamento dos indivíduos mais populares do grupo (Dijkstra, Lindenberg, \& Veenstra, 2008). Além da importância da norma do grupo, a apresentação de comportamentos que exprimem habilidades sociais, intelectuais e acadêmicas, ao se equilibrar com a agressividade, evita que esta prediga a rejeição pelos colegas (Nelson, Robinson, \& Hart, 2005; Newcomb et al., 1993; Orue \& Calvete, 2011). Além desse fator, destaca-se que, ao se utilizar de respostas agressivas em determinadas circunstâncias - diante de provocações ou em defesa de sua honra e de colegas, por exemplo - a criança não tem seu relacionamento com os colegas prejudicado, pois estes entendem a agressão como justificada e, por isso, ela é tolerada e encorajada pelo grupo.

Neste contexto, compreende-se que uma criança cujo padrão comportamental se diferencia do que é apresentado e socialmente aceito pelo seu grupo terá maiores chances de ser rejeitada pelos colegas. No caso do filme, Charlie, caracterizado pelo seu retraimento e sua passividade, possui um repertório comportamental diferente daquele apresentado pelos seus colegas de escola, repertório o qual seria esperado segundo a norma social ali presente, na qual inclusive comportamentos de caçoar os alunos são socialmente aceitos, por exemplo. Partindo deste ponto, pode-se conjecturar que se trata de um caso de uma criança que pode ter sido rejeitada em função, entre outros fatores, da apresentação de comportamentos internalizantes, e não de comportamentos externalizantes.

É importante ressaltar que a rejeição pelos pares se associa a problemas em curto e longo prazo, independentemente da presença ou não de comportamentos agressivos. Por exemplo, tanto as crianças rejeitadas agressivas como as não agressivas podem apresentar sintomatologia depressiva decorrentes de seus status 
social (Hecht, Inderbitzen, \& Bukowski, 1998). No entanto, o caráter dessa sintomatologia vai depender da natureza das dificuldades na relação com os pares, ou seja, enquanto, para as crianças agressivas, a depressão se associa a problemas interpessoais, no caso das crianças não agressivas, ela está mais associada a baixos níveis de interações sociais (Hecht et al., 1998). É possível estabelecer um paralelo entre este dado e o momento do filme no qual, diante do afastamento de seus amigos, os comportamentos depressivos de Charlie aparecem, o que é expresso pelo próprio personagem, ao escrever "não vejo meus amigos há duas semanas. Estou começando a ficar mal de novo".

\subsection{Rejeição e o papel do professor}

Há uma linha de pesquisa dentro do tema da rejeição entre pares que tem investigado a influência do professor no status social da criança (Farmer, Lines, \& Hamm, 2011; Maulana, Opdenakker, Stroet, \& Bosker, 2013; Wubbels, Brekelmans, den Brok, Wijsman, Mainhard, \& van Tartwijk, 2014). Segundo Luckner e Pianta (2011), existem três maneiras a partir das quais o professor pode exercer influência nas relações que os alunos estabelecem com seus pares, a saber, a organização da sala de aula, o apoio instrucional e o apoio emocional. O domínio da organização é constituído pelas interações professoraluno envolvidas no manejo do tempo, do comportamento e da atenção na sala de aula (Luckner \& Pianta, 2011). O modo como esse domínio pode se relacionar com as experiências com os colegas reside na influência indireta das oportunidades para interações entre pares e do apoio ao desenvolvimento de habilidades necessárias para a emissão de respostas adequadas diante dos comportamentos dos colegas.

O apoio instrucional, por sua vez, inclui a qualidade das interações instrucionais entre professores e alunos no que se refere à riqueza da instrução e ao feedback fornecido ao aluno (Luckner \& Pianta, 2011). $\mathrm{O}$ apoio instrucional pode influenciar indiretamente o comportamento dos pares a partir de oportunidades fornecidas às crianças para que trabalhem diretamente com os colegas tendo em vista um objetivo em comum. Por meio desse apoio instrucional, os professores também possibilitariam os desenvolvimentos cognitivo e linguístico das crianças, que estão relacionados à interação entre pares. Além desses aspectos, pode-se mencionar o fato de que as instruções modelam o tipo e a frequência das interações entre as crianças e podem afetar a maneira como as regras sociais implícitas na sala de aula favorecerão a inclusão ou a exclusão (Donohue, Perry, \& Weinstein, 2003). Por exemplo, o ensino centrado na criança, quando comparado àquele que é direcionado pelo professor ou que se baseia 
em instruções de habilidades básicas, pode tornar menos propensa a rejeição dos alunos pelos seus colegas.

Apesar das evidências relativas à influência do apoio instrucional e da organização de sala de aula serem mistas, Luckner e Pianta (2011) afirmam que são consistentes entre os estudos as evidências de que a qualidade do apoio emocional nas interações professor-aluno nos anos iniciais do Ensino Fundamental se relaciona ao comportamento dos colegas das crianças. $O$ apoio emocional se refere à conexão emocional entre o professor e seus alunos e à consciência e responsividade do professor em relação ao funcionamento social e emocional dos alunos. Luckner e Pianta (2011) defendem que, a partir desse apoio emocional, os professores fornecem às crianças modelos de habilidade relacionais e de atitudes que influenciam 0 modo como as crianças agirão em sua relação com os pares. Além disso, a partir dessas interações, o professor proveria uma base segura para o aluno assumir riscos em suas interações com os colegas.

De fato, pesquisas apontam que a relação professor-aluno, quando positiva e responsiva, é importante para evitar a rejeição pelos pares, pois atua como um recurso compensatório no ajustamento social tanto de crianças agressivas, quanto de crianças com padrão mais retraído e ansioso (Kiuru et al., 2012). Não somente isto, mas se trata de uma relação que auxilia a evitar os impactos negativos dos riscos sociais e acadêmicos da rejeição, visto que contribui para uma melhor autorregulação da criança, melhores habilidades sociais e um melhor discernimento emocional (Kiuru et al., 2012).

Outro fator bastante abordado na literatura e também relacionado ao modo como o professor pode interferir nas relações entre as crianças se refere às evidências de que uma baixa preferência do professor em relação a determinados alunos está relacionada à rejeição pelos pares (Kiuru et al., 2012). Neste contexto, a qualidade das relações professor-aluno, que pode ser marcada, por exemplo, pelo favorecimento daqueles que apresentam um alto desempenho acadêmico, reflete nas relações que as crianças apresentam entre si em sala de aula (Donohue et al., 2003; Spilt, Lier, Leflot, Onghena, \& Colpin, 2014). Vale ressaltar que são poucos os estudos que indicam que a rejeição pelos pares leva à baixa preferência do professor, sendo que a maioria das pesquisas traz resultados que apontam para o caminho inverso, ou seja, que a baixa preferência do professor leva à rejeição social (Kiuru et al., 2012).

A partir do exposto neste tópico, compreende-se que, além das características individuais da criança, o contexto da sala de aula também pode influenciar no desenvolvimento da rejeição, sendo que o professor exerce um papel consideravelmente importante (Donohue et al., 2003; Spilt et al., 2014). Tendo em vista esta relevância, optou-se por abordar essa questão no presente artigo, ainda que a 
relação do filme com este tema seja mais frágil que a relação com os temas acima abordados, dado que não é retratado abertamente o modo como Charlie se relacionou com seus professores durante sua infância e que é apresentado diretamente somente um relacionamento específico com um único professor, Mr. Anderson, estabelecido durante a adolescência.

A interação com Mr. Anderson se mostra positiva, podendo-se levantar a possibilidade de que foi um fator que contribuiu naquele momento para que não ocorressem maiores desdobramentos negativos associados à condição de rejeição do personagem, abrindo espaço inclusive para que ele desenvolvesse suas habilidades acadêmicas, por meio da leitura dos livros sugeridos pelo professor e de seu empenho na escrita. Por outro lado, pela fala do protagonista referindo-se a esse professor como sendo o melhor que já teve, pode-se levantar a hipótese de que, durante a infância, faltaram ao personagem interações positivas com seus professores que poderiam ter auxiliado na proteção contra aspectos que o tornaram rejeitados pelos seus pares - tal como seu padrão comportamental retraído - e, mais do que isso, contra uma série de dificuldades retratadas ao longo do filme decorrentes de sua condição de rejeição.

\section{Considerações finais}

Este artigo teve por objetivo relacionar a rejeição entre pares com a amizade estabelecida com colegas, os comportamentos agressivos que podem ser apresentados pela criança rejeitada, suas habilidades sociais e o papel do professor neste contexto, utilizando, paralelamente, o filme The perks of being a wallflower como um recurso didático para exemplificar os aspectos descritos. A rejeição entre pares, entendida como uma categoria sociométrica em que prevalecem as nomeações negativas sobre as positivas, é um problema corrente na infância e pode contribuir para a ocorrência de delinquência juvenil, uso de drogas, evasão escolar e depressão. A experiência de rejeição pode iniciar ou exacerbar dificuldades de ajustamento social da criança e esse status pode se manter ao longo de vários anos escolares. Alguns padrões comportamentais e 0 contexto social ao qual a criança pertence, principalmente as interações estabelecidas no ambiente escolar, parecem ter importante influência na rejeição.

Sem perder de vista o fato do uso do filme como recurso didático para a apresentação do tema se configurar como uma extrapolação dos dados por ele fornecidos, inferiu-se o status de rejeição de Charlie em função de seu estilo comportamental e das interações estabelecidas com seus pares. Partindo deste ponto, relacionaram-se 
as características apresentadas pelo personagem principal e as variáveis contextuais à sua condição de rejeição.

Em termos de suas amizades, compreende-se que Charlie encontrou nos amigos uma proteção contra alguns dos riscos decorrentes da rejeição pelos colegas, atenuando a emissão de problemas de comportamentos internalizantes, traduzidos pela sua condição de solidão e pelos comportamentos depressivos. Ao mesmo tempo, no entanto, essa associação acirrou comportamentos desviantes, o que pôde ser observado de forma mais clara nas cenas que retratam o uso de álcool e de substâncias psicoativas, o qual o filme sugere não ter ocorrido antes da criação do vínculo com o grupo de amigos.

Apesar de a literatura trazer grande ênfase ao comportamento agressivo das crianças como preditor de rejeição, essa relação não é válida em todos os casos, como exemplificado no caso do filme. Charlie, ainda que não tenha se engajado, durante a infância, em comportamentos de agressividade, adquire a condição de rejeição, possivelmente pelo fato de seu estilo comportamental internalizante ser contrário às normas sociais de seu contexto escolar e, por isso, se configurar como um repertório não aceito pelos demais alunos.

Com relação às habilidades sociais do protagonista, pode-se notar que sua carência no personagem leva a uma espiral de restrições de atividades sociais, tanto no contexto geral da escola quanto no seu novo círculo de amigos, restrições estas que ampliam seus problemas de relacionamento e de comportamentos internalizantes, uma vez que o priva de passar por importantes experiências de socialização, ter oportunidades de desenvolver suas habilidades sociais e adquirir importantes fontes de apoio social.

Em sala de aula, o professor exerce uma importante influência no relacionamento entre os alunos e pode ajudar a proteger o aluno tanto contra a rejeição, quanto contra os desdobramentos negativos a ela associados. A despeito do fato da relação do filme com este tema ser mais frágil, considerou-se a relevância do papel do professor no contexto da rejeição para abordar a questão. Sendo assim, discorreu-se sobre a possibilidade de que a interação com Mr. Anderson contribuiu para que não ocorressem maiores prejuízos associados à condição de rejeição de Charlie. Ainda, sugeriu-se que faltou ao personagem, no passado, interações positivas com seus professores, as quais poderiam ter auxiliado na proteção contra aspectos que o tornaram rejeitado pelos seus pares, assim como contra uma série de dificuldades retratadas ao longo do filme decorrentes de sua condição de rejeição.

Ainda que se trate de um personagem cujo comportamento está relacionado a uma série de outras variáveis, além da rejeição social, Charlie pode ser retratado como um indivíduo que apresenta problemas durante a adolescência possivelmente decorrentes em parte de uma condição social de rejeição vivida na infância, mas cuja 
visibilidade foi baixa em função de seu comportamento caracteristicamente internalizante, o qual pode também ter sido agravado pelo seu, assim inferido, status sociométrico. É possível que seu status tivesse sido outro caso Charlie apresentasse um maior repertório de habilidades sociais construído a partir de relações positivas com professores que pudessem lhe servir como modelo, por exemplo. Pode-se, ainda, levantar a hipótese de que, se o personagem tivesse sido agressivo durante a infância, suas dificuldades comportamentais teriam recebido uma maior atenção e, assim, ele poderia ter sido submetido a alguma intervenção psicológica, a qual visasse ao desenvolvimento de comportamentos pró-sociais que evitassem a ocorrência dos problemas decorrentes da rejeição.

A partir dos pontos levantados acerca das características e consequências da rejeição pelos pares, o presente artigo possibilitou lançar luz sobre a relevância de estudos de caráter sociométrico no contexto escolar, principalmente em cenário nacional, cuja literatura ainda é escassa. Estes estudos podem trazer importantes contribuições para o delineamento de intervenções preventivas junto aos professores e aos próprios alunos, tendo como foco a promoção e o fortalecimento, tanto nos educadores quanto nos estudantes, de um repertório comportamental mais favorável ao adequado desenvolvimento das crianças e dos adolescentes.

Destaca-se que as relações, estabelecidas no presente trabalho, entre as cenas do filme e os diferentes aspectos da rejeição pelos pares indicam a possibilidade do uso do longa-metragem para ampliar a visibilidade do tema no meio acadêmico, com o intuito de incentivar mais pesquisas relacionadas a esta linha investigativa. Não apenas isto, mas o uso das relações apresentadas ao longo do presente artigo também pode trazer benefícios em termos da ampliação do conhecimento que as pessoas responsáveis pelo desenvolvimento saudável da população infanto-juvenil têm acerca dos prejuízos associados à rejeição e das formas de preveni-la.

\section{Referências}

Argyle, M., \& Furnham, A. (1981). Social situations. Cambridge, UK: Cambridge University Press.

Bagwell, C. L., \& Schmidt, M. E. (2013). Friendships in childhood and adolescence. Guilford Press.

Bierman, K. L. (2004). Peer rejection: Developmental processes and intervention strategies. Guilford Press.

Caldarella, P., \& Merrell, K. W. (1997). Common dimensions of social skills of children and adolescents: A taxonomy of positive behaviors. School Psychology Review, 26(2), 264-278. 
Castro, R. E. F. d., Melo, M. H. d. S., \& Silvares, E. F. d. M. (2003). O julgamento de pares de crianças com dificuldades interativas após um modelo ampliado de intervenção. Psicologia: Reflexão e Crítica, 16(2), 309-318.

Cillessen, A. H. (2009). Sociometric methods. In: K. H. Rubin, W. M. Bukowski, Brett, Laursen (Orgs.). Handbook of peer interactions, relationships, and groups. Social, emotional, and personality development in context (pp. 82-99). New York, NY, US: Guilford Press.

Cillessen, A. H., Bukowski, W. M., \& Haselager, G. J. (2000). Stability of sociometric categories. In: A. H. N. C. a. W. M. Bukowski (Ed.), Recent advances in the measurement of acceptance and rejection in the peer system (Vol. 99, pp. 75-93). San Francisco: Jossey-Bass. doi: 10.1002/cd.23220008807

Coie, J. D., Dodge, K. A., \& Coppotelli, H. (1982). Dimensions and types of social status: A cross-age perspective. Developmental psychology, 18(4), 557-570. doi: 10.1037/0012-1649.19.2.224

Del Prette, Z. A. P., \& Del Prette, A. (2005). Psicologia das habilidades sociais na infância: teoria e prática. Petrópolis: Vozes.

Del Prette, Z. A. P., \& Del Prette, A. (1999). Psicologia das habilidades sociais: terapia e educação. Petrópolis: Vozes.

Del Prette, Z. A. P., \& Del Prette, A. (2010). Habilidades sociais e análise do comportamento: Proximidade histórica e atualidades. Revista perspectivas, 1(2), 104-115. doi: 104115_RP_2010_01_02

Del Prette, A., \& Del Prette, Z. A. P. (2013). Habilidades sociais: Intervenções efetivas em grupo. São Paulo: Casa do Psicólogo.

Dijkstra, J. K., Lindenberg, S., \& Veenstra, R. (2007). Same-gender and cross-gender peer acceptance and peer rejection and their relation to bullying and helping among preadolescents: Comparing predictions from gender-homophily and goalframing approaches. Developmental Psychology, 43(6), 1377. doi: $10.1037 / 0012-1649.43 .6 .1377$

Dijkstra, J. K., Lindenberg, S., \& Veenstra, R. (2008). Beyond the class norm: Bullying behavior of popular adolescents and its relation to peer acceptance and rejection. Journal of Abnormal Child Psychology, 36(8), 1289-1299. doi: 10.1007/s10802-0089251-7

Donohue, K. M., Perry, K. E., \& Weinstein, R. S. (2003). Teachers' classroom practices and children's rejection by their peers. Journal of Applied Developmental Psychology, 24(1), 91-118. doi: 10.1016/S0193-3973(03)00026-1

Farias, A. K. d., \& Ribeiro, M. R. (2007). Skinner vai ao Cinema. Santo André, SP: ESETec. 
Farmer, T. W., Lines, M. M., \& Hamm, J. V. (2011). Revealing the invisible hand: The role of teachers in children's peer experiences. Journal of Applied Developmental Psychology, 32(5), 247-256.

Feldman, S., \& Downey, G. (1994). Rejection sensitivity as a mediator of the impact of childhood exposure to family violence on adult attachment behavior. Development and Psychopathology, 6(1), 231-247. doi: 10.1017/S0954579400005976

Fink, E., Rosnay, M., Peterson, C., \& Slaughter, V. (2013). Validation of the Peer Social Maturity Scale for Assessing Children's Social Skills. Infant and Child Development, 22(5), 539-552.

Hecht, D. B., Inderbitzen, H. M., \& Bukowski, A. L. (1998). The relationship between peer status and depressive symptoms in children and adolescents. Journal of Abnormal Child Psychology, 26(2), 153-160. doi: 10.1023/A:1022626023239

Hodges, E. V., Boivin, M., Vitaro, F., \& Bukowski, W. M. (1999). The power of friendship: protection against an escalating cycle of peer victimization. Developmental Psychology, 35(1), 94-101. doi: 10.1037/0012-1649.35.1.94

Hodges, E. V., Malone, M. J., \& Perry, D. G. (1997). Individual risk and social risk as interacting determinants of victimization in the peer group. Developmental Psychology, 33(6), 1032-1039.

Kiuru, N., Poikkeus, A. M., Lerkkanen, M. K., Pakarinen, E., Siekkinen, M., Ahonen, T., \& Nurmi, J. E. (2012). Teacherperceived supportive classroom climate protects against detrimental impact of reading disability risk on peer rejection. Learning and Instruction, 22(5), 331-339. doi: 10.1016/j.learninstruc. 2011.12.003

Lansford, J. E., Dodge, K. A., Fontaine, R. G., Bates, J. E., \& Pettit, G. S. (2014). Peer rejection, affiliation with deviant peers, delinquency, and risky sexual behavior. Journal of Youth and Adolescence, 43(10), 1742-1751.

Laursen, B., Bukowski, W. M., Aunola, K., \& Nurmi, J. E. (2007). Friendship moderates prospective associations between social isolation and adjustment problems in young children. Child Development, 78(4), 1395-1404. doi: 10.1111/j.14678624.2007.01072.x

Leary, M. R., Twenge, J. M., \& Quinlivan, E. (2006). Interpersonal rejection as a determinant of anger and aggression. Personality and Social Psychology Review, 10(2), 111-132. doi: 10.1207/s15327957pspr1002_2

Leflot, G., van Lier, P. A., Onghena, P., \& Colpin, H. (2013). The role of children's on-task behavior in the prevention of aggressive behavior development and peer rejection: A randomized controlled study of the Good Behavior Game in Belgian 
elementary classrooms. Journal of School Psychology, 51(2), 187-199. doi: 10.1016/j.jsp.2012.12.006

Luckner, A. E., \& Pianta, R. C. (2011). Teacher-student interactions in fifth grade classrooms: Relations with children's peer behavior. Journal of Applied Developmental Psychology, 32(5), 257-266. doi: 10.1016/j.appdev.2011.02.010

López, E. E., Olaizola, J. H., Ferrer, B. M., \& Ochoa, G. M. (2006). Aggressive and nonaggressive rejected students: An analysis of their differences. Psychology in the Schools, 43(3), 387-400. doi: 10.1002/pits.20152

Maulana, R., Opdenakker, M. C., Stroet, K., \& Bosker, R. (2013). Changes in teachers' involvement versus rejection and links with academic motivation during the first year of secondary education: A multilevel growth curve analysis. Journal of Youth and Adolescence, 42(9), 1348-1371.

McFadyen-Ketchum, S., \& Dodge, K. A. (1998). Problems in social relationships. In: E. J. M. a. B. A. Barkley (Ed.), Treatment of childhood disorders (2 ed., pp. 338-365). New York: Guilford Press.

Melo, M. H. S. (2006). Programa de treinamento para promover competência social em crianças e seus professores no ambiente escolar (Relatório de Pesquisa (Pós-doutorado) ed.). São Paulo: Departamento de Psicologia Clínica, Universidade de São Paulo.

Mikami, A. Y., Lerner, M. D., \& Lun, J. (2010). Social context influences on children's rejection by their peers. Child Development Perspectives, 4(2), 123-130. doi: 10.1111/j.1750-8606.2010.00130.x

Mikami, A. Y., \& Normand, S. (2015). The Importance of Social Contextual Factors in Peer Relationships of Children with ADHD. Current Developmental Disorders Reports, 1-8, 30-37.

Monahan, K. C., Steinberg, L., \& Cauffman, E. (2009). Affiliation with antisocial peers, susceptibility to peer influence, and antisocial behavior during the transition to adulthood. Developmental Psychology, 45(6), 1520-1530. doi: 10.1037/a0017417

Mrug, S., Molina, B. S., Hoza, B., Gerdes, A. C., Hinshaw, S. P., Hechtman, L., \& Arnold, L. E. (2012). Peer rejection and friendships in children with attention-deficit/hyperactivity disorder: contributions to long-term outcomes. Journal of Abnormal Child Psychology, 40(6), 1013-1026. doi: 10.1007/s10802-012-9610-2

Nelson, D. A., Robinson, C. C., \& Hart, C. H. (2005). Relational and physical aggression of preschool-age children: Peer status linkages across informants. Early Education \& Development, 16(2), 115-140. doi: 10.1207/s15566935eed1602_2

Newcomb, A. F., Bukowski, W. M., \& Pattee, L. (1993). Children's peer relations: a meta-analytic review of popular, rejected, 
neglected, controversial, and average sociometric status. Psychological Bulletin, 113(1), 99-128.

Orue, I., \& Calvete, E. (2011). Reciprocal relationships between sociometric indices of social status and aggressive behavior in children Gender differences. Journal of Social and Personal Relationships, 28(7), 963-982. doi: $10.1177 / 0265407510397982$

Oxford English Dictionary (1989). Oxford, UK: Oxford University Press.

Parker, R. J. (2011). Closeness and Conflict in Children's Friendships: Relations with Friendship Stability, Adjustment and Sociometric Status (Doctoral dissertation, University of Ottawa).

Parker, J. G., \& Asher, S. R. (1993). Friendship and friendship quality in middle childhood: Links with peer group acceptance and feelings of loneliness and social dissatisfaction. Developmental Psychology, 29(4), 611-621. doi: 10.1037/0012-1649.29.4.611

Rudolph, K. D., Lansford, J. E., Agoston, A. M., Sugimura, N., Schwartz, D., Dodge, K. A., Bates, J. E. (2014). Peer victimization and social alienation: predicting deviant peer affiliation in middle school. Child Development, 85(1), 124-139. doi: $10.1111 /$ cdev.12112

Salazar, S., Boivin, M., Vitaro, F., Cantin, S., Forget-Dubois, N., Brendgen, M., \& Tremblay, R. (2015). Friendships and deviancy training in young children. Journal of Aggression, Conflict and Peace Research, 7(2), 112-123.

Scott, J. P. (2015). Anger and aggression: Links to social (mal) adjustment in early adolescence (Doctoral dissertation, ST. John's University, School of Education and Human Services).

Spilt, J. L., Lier, P. A., Leflot, G., Onghena, P., \& Colpin, H. (2014). Children's Social Self-Concept and Internalizing Problems: The Influence of Peers and Teachers. Child Development, 85(3), 1248-1256.

Silvares, E. F. M., \& Melo, M. H. S. (2010). Rejeição Infantil: o papel dos colegas de escola e professores. In: M. P. R. d. Souza (Ed.), Ouvindo crianças na escola (1 ed., pp. 102-112). Itatiba, SP: Casa do Psicólogo.

Veenstra, R., Lindenberg, S., Munniksma, A., \& Dijkstra, J. K. (2010). The complex relation between bullying, victimization, acceptance, and rejection: Giving special attention to status, affection, and sex differences. Child Development, 81(2), 480486. doi: 10.1111/j.1467-8624.2009.01411.x

Wubbels, T. H., Brekelmans, J. M. G., den Brok, P. J., Wijsman, L., Mainhard, T., \& van Tartwijk, J. W. F. (2014). Teacher-student relationships and classroom management. In: E. T. Emmer \& E. Sarbonie (Eds.), Handbook of classroom management (2nd edition). London: Taylor \& Francis. 


\section{Endereço para correspondência \\ Sarah Izbicki}

Universidade de São Paulo

Instituto de Psicologia

Av. Prof. Mello Moraes, 1721, bl. F, sala 19, CEP 05508-030, São Paulo - SP, Brasil

Endereço eletrônico: sarah.izbicki@usp.br

Luiza Chagas Brandão

Universidade de São Paulo

Instituto de Psicologia

Av. Prof. Mello Moraes, 1721, bl. F, sala 19, CEP 05508-030, São Paulo - SP, Brasil

Endereço eletrônico: luiza.brandao@usp.br

\section{Lígia Mosolino de Carvalho}

Universidade de São Paulo

Instituto de Psicologia

Av. Prof. Mello Moraes, 1721, bl. F, sala 19, CEP 05508-030, São Paulo - SP, Brasil Endereço eletrônico: ligia.mosolino.carvalho@usp.br

\section{Márcia Helena da Silva Melo}

Universidade de São Paulo

Instituto de Psicologia

Av. Prof. Mello Moraes, 1721, bl. F, sala 19, CEP 05508-030, São Paulo - SP, Brasil

Endereço eletrônico: mmelo@usp.br

Recebido em: 20/08/2014

Reformulado em: 07/05/2015

Aceito para publicação em: 11/05/2015

\section{Notas}

* Psicóloga, Especialista em Terapia Comportamental e Mestre em Psicologia Clínica pelo Instituto de Psicologia da Universidade de São Paulo.

** Psicóloga e Mestranda em Psicologia Clínica pelo Instituto de Psicologia da Universidade de São Paulo.

*** Psicóloga e Mestranda em Psicologia Experimental pelo Instituto de Psicologia da Universidade de São Paulo.

**** Psicóloga pela Universidade Federal do Pará, Mestre e Doutora em Psicologia pelo Instituto de Psicologia da Universidade de São Paulo, Prof.a Dra. do Departamento de Psicologia Clínica do Instituto de Psicologia da Universidade de São Paulo.

${ }^{1}$ A vitimização pode assumir a forma de abuso físico (p. ex. bater), verbal (p. ex. xingamento), ou relacional (p. ex. ostracismo social) (Rudolph et al., 2014). 